\title{
Reachability on a region bounded by two attached squares
}

\author{
Ali Mohades \\ mohades@cic.aku.ac.ir \\ AmirKabir University of Tech., Math. and Computer Sc. Dept.
}

\author{
Mohammadreza Razzazi \\ razzazi@ce.aku.ac.ir \\ AmirKabir University of Tech., Computer Eng. Dept.
}

\begin{abstract}
This paper considers a region bounded by two attached squares and a linkage confined within it. By introducing a new movement called mot, presents a quadratic time algorithm for reaching a point inside the region by the end of the linkage. It is shown that the algorithm works when a certain condition is satisfied.
\end{abstract}

keywords: Multi-link arm, reachability, motion planning, concave region, robot arms.

\section{Introduction}

This paper considers the movement of a linkage in a two-dimensional bounded region and introduces a new algorithm to reach a given point by the end of the linkage. The region considered is the one obtained by two attached squares.

Several papers have been written on reachability problems mainly, on convex region. Hopcroft, Joseph and Whitesides in [1] studied the reconfiguration and reachability problems for a linkage. In [2], they gave a polynomial time algorithm for moving a linkage confined within a circle from one given configuration to another, and proved that the reachability problem for a planar arm constrained by an arbitrary polygon, is NP-hard. Joseph and Plantings [3] proved that the reachability problem for a chain moving within a certain non-convex constraining environment is PSPACE hard.

In [4] and [5], Kantabutra presented a linear time algorithm for reconfiguring certain chains inside squares. He considered an unanchored n-linkage robot arm confined inside a square with side length at least as long as the longest arm link and found a necessary and sufficient condition for reachability in this square. His algorithm requires $\mathrm{O}(\mathrm{n})$ time.

This paper extends the previous results by providing a quadratic time algorithm to solve the reachability problem in a special concave region. The 
region is bounded by the union of two squares attached via one edge. In the next section of the paper some preliminaries and useful definitions are given. In section 3 a new movement, by which a linkage moves in a concave corner is formulated and finally in section 4 present the reachability algorithm and the related properties are presented.

\section{Preliminaries}

An $n$-linkage $\Gamma[0,1, \ldots \mathrm{n}]$ is a collection of $\mathrm{n}$ rigid rods or links, $\left\{A_{i-1} A_{i}\right\}_{i=1, \ldots n}$, consecutively joined together at their end points, about which they may rotate freely. Links may cross over one another and none of end points of the linkage are fixed.

We denote the length of links of $\Gamma[0,1, \ldots \mathrm{n}]$ by $l_{1}, l_{2}, \ldots l_{n}$, where $l_{i}$ is the length of link with end points $A_{i-1}$ and $A_{i}$ and $\|\Gamma\|=\max _{1 \leq i \leq n} l_{i}$. For $1 \leq i \leq n-1$ the angle obtained by turning clockwise about $A_{i}$ from $A_{i-1}$ to $A_{i+1}$ is denoted by $\alpha_{i}$. We say that a linkage $\Gamma$ is bounded by $b$ if $\|\Gamma\|<$ $\mathrm{b}$, i.e no link has a length greater than or equal to $\mathrm{b}$.

For a region $\mathrm{P}$, by Reaching a given point $p \in P$ by $A_{n}$, the end point of $\Gamma$, we mean $\Gamma$ can move within $\mathrm{P}$ from its given initial position to a final position so that $A_{n}$ reaches p.

For a linkage $\Gamma$ confined inside a convex region $\mathrm{P}$ with boundary denoted by $\partial P$, we define two special configurations as follows (Figure 1):

We say that $\Gamma$ is in Rim Normal Form (denoted RNF), if all its joints lie on $\partial \mathrm{P}$.

We say that $\Gamma$ is in Ordered Normal Form (denoted ONF), if:

1. $\Gamma$ is in RNF.

2. Moving from $A_{0}$ toward $A_{n}$ along $\Gamma$ is always either clockwise or counterclockwise around the boundary polygon.

Algorithms for the reconfiguration of an n-linkage usually break up the motions for the whole reconfiguration into simple motions, in which only a few joints are moved simultaneously (see [2], [6] and [7]). We allow the following type of simple motions:

- No angle at joints changes, but the linkage may translate and rotate as a rigid object.

- At most four angles change simultaneously and the other joints do not change their positions.

\section{Movement in a concave environment}

In this section we introduce a new movement for a linkage to reach a point inside a certain concave region. 


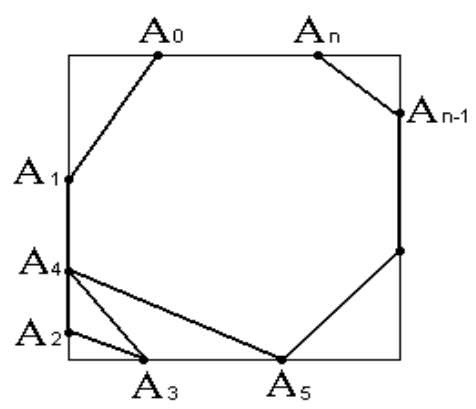

(a)

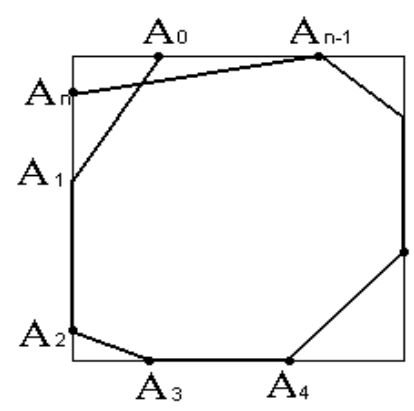

(b)

Figure 1: An n-linkage in (a): Rim Normal Form, (b): Ordered Normal Form.

Theorem 1. Suppose that $\mathrm{S}$ is a region where its boundary polygon $\partial S$, is a square with side length $\mathrm{s}, \Gamma[0,1, \ldots n]$ is an n-linkage confined within $\mathrm{S}$ and $\|\Gamma\|<\mathrm{s}$. Then $\Gamma$ can be brought to ONF using $\mathrm{O}(\mathrm{n})$ simple motions.

Proof: See [5].

Lemma 2. If $\partial S$, the boundary polygon of the region $\mathrm{S}$, is a square with side length $\mathrm{s}$ and $\Gamma[0,1, \ldots n]$ is an n-linkage with $\|\Gamma\|<\mathrm{s}$ confined within $\mathrm{S}$, initially in ONF. Then any joint of $\Gamma$ can be moved along $\partial S$ in either direction, in such a manner that the linkage always remain in ONF. This can be done with $\mathrm{O}(\mathrm{n})$ simple motions.

\section{Proof: See [5].}

To understand our new movement, it helps to first consider a special case of 2-linkage $\Gamma[1,2,3]$ consisting of joints $A_{1}, A_{2}$ and $A_{3}$. We define a movement for $\Gamma[1,2,3]$ from its initial configuration to a specified final configuration in which, $A_{1}$ gets the position of $A_{2}$, and $A_{3}$ moves forward in a given path (Figure 2).

Unless otherwise specified, by $\angle A_{1} A_{2} A_{3}\left(\angle \gamma_{1} \gamma_{2}\right.$, which $\gamma_{1}$ and $\gamma_{2}$ are two crossing line segments), we mean the angle obtained by turning clockwise from $A_{1}$ to $A_{3}$ about $A_{2}$ (from $\gamma_{1}$ to $\gamma_{2}$ ).

Circumstances: Consider two line segments $\gamma_{1}$ and $\gamma_{2}$ which intersect at $\mathrm{q}$ and $\angle \gamma_{1} \gamma_{2}$ is in $[\pi, 2 \pi]$. Let $\rho$ be the line segment which starts at $\mathrm{q}$ and divides the angle $\angle \gamma_{1} \gamma_{2}$ into two angles $\angle \gamma_{1} \rho$ and $\angle \rho \gamma_{2}$ in such a way that $\angle \gamma_{1} \rho$ is in $[\pi / 2, \pi]$. Initial configuration of $\Gamma[1,2,3]$ is defined as follows: Let $A_{1}$ be at point $\mathrm{p}$ on line segment $\gamma_{1}, A_{2}$ at $\mathrm{q}$ and $A_{3}$ at point $\mathrm{r}$ on line segment $\gamma_{2}$ (Figure 2-a). By this assumption we can define our movement in a concave region. 


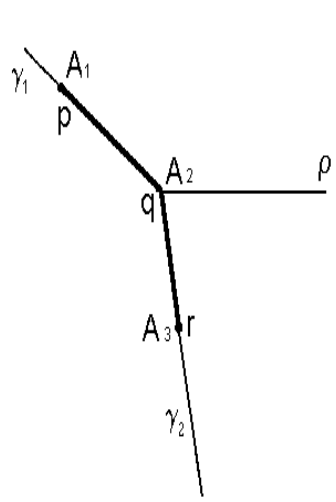

(a)

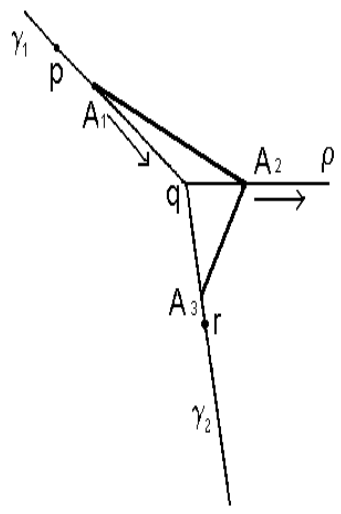

(b)

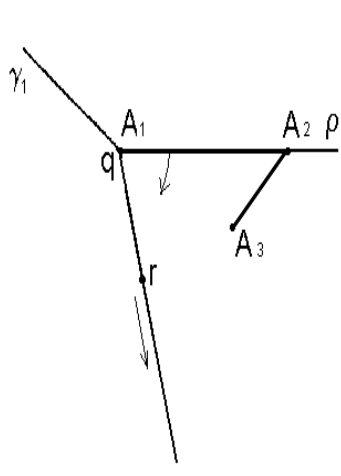

(c)

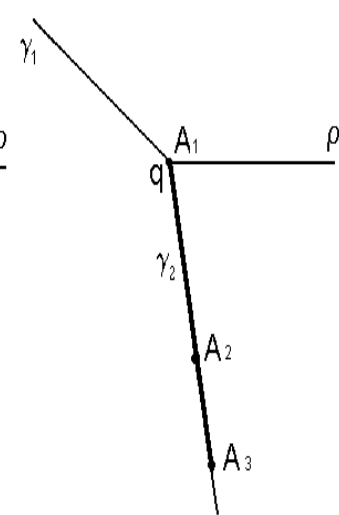

(d)

Figure 2: (a): Initial configuration of $\Gamma[1,2,3]$, (b): middle-jointup $\left(A_{1}, A_{2}, A_{3}, \rho\right)$ motion, (c): front-link-forward $\left(A_{1}, A_{2}, A_{3}, \rho\right)$ motion, (d): final configuration of $\Gamma[1,2,3]$.

Definition 3. The $\operatorname{mot}\left(A_{1}, A_{2}, A_{3}, \rho\right)$ movement changes the initial configuration of $\Gamma[1,2,3]$ to a final configuration by which $\Gamma$ lies on $\gamma_{2}$. This is done by two consecutive motions:

- Middle-joint-up $\left(A_{1}, A_{2}, A_{3}, \rho\right)$ : moves $A_{2}$ along $\rho$ away from q until $A_{1}$ reaches q. During the movement $A_{1}$ remains on $\gamma_{1}$, and $A_{3}$ remains on $\gamma_{2}$ as much as possible.

- Front-link-forward $\left(A_{1}, A_{2}, A_{3}, \rho\right)$ : fixes $A_{1}$ at q and brings down $A_{3}$ on $\gamma_{2}$ (if not already there). To straighten $\Gamma$, it moves $A_{3}$ along $\gamma_{2}$ away from $q$.

We show the $\operatorname{mot}\left(A_{1}, A_{2}, A_{3}, \rho\right)$ movement can be done in finite number of simple motions.

Assume $\Gamma$ is in the initial configuration. We show how each of the middlejoint-up motion and front-link-forward motion is done in finite number of simple motions.

Middle-joint-up $\left(A_{1}, A_{2}, A_{3}, \rho\right)$ :

Move $A_{2}$ along $\rho$ away from q (Figure 2-b). If $\angle \rho \gamma_{2} \geq \pi / 2$, during the movement, $A_{1}$ and $A_{3}$ approach q, while staying on lines $\gamma_{1}$ and $\gamma_{2}$ respectively.

If $\angle \rho \gamma_{2}<\pi / 2$, during the movement, $A_{3}$ moves away from $\mathrm{q}$ and it is possible that $A_{2} A_{3}$ becomes perpendicular to $\gamma_{2}$. If this happens, first turn $A_{2} A_{3}$ about $A_{2}$ until q $A_{2} A_{3}$ folds, then if needed, move $A_{2} A_{3}$ along $\rho$ away from q in a way that $\alpha_{2}$ increases until $A_{1} A_{2} A_{3}$ folds and $A_{1}$ reaches q. This requires a finite number of simple motions. 
Front-link-forward $\left(A_{1}, A_{2}, A_{3}, \rho\right)$ :

If during middle-joint-up motion $A_{1}$ reaches q first, for applying front-linkforward motion, it is enough to keep $A_{1}$ at q fixed, and move $A_{3}$ along $\gamma_{2}$ until $\Gamma$ straightens.

If $A_{3}$ reaches q first and $A_{1}$ arrives later, for applying front-link-forward motion, turn $A_{2} A_{3}$ about $A_{2}$ in a way that $\alpha_{2}$ decreases, until $A_{3}$ hits $\gamma_{2}$ or $\alpha_{2}=3 \pi / 2$. If $\alpha_{2}=3 \pi / 2$ before $A_{3}$ hits $\gamma_{2}$, rotate $\Gamma$ about $A_{1}$ in a way that $\angle A_{2} A_{1} r$ decreases until $A_{3}$ reaches $\gamma_{2}$, then keep $A_{1}$ fixed at q and move $A_{3}$ along $\gamma_{2}$ away from q so that $\Gamma$ straightens. This requires a finite number of simple motions (Figure 2-c).

If $A_{3}$ hits $\gamma_{2}$ first, keep $A_{1}$ fixed at q and move $A_{3}$ along $\gamma_{2}$ away from q so that $\Gamma$ straightens.

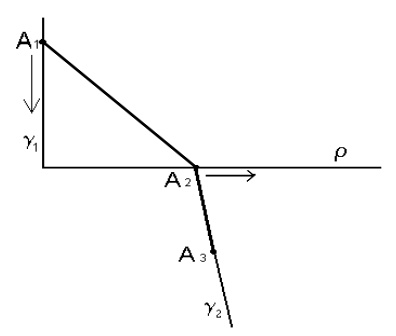

Figure 3: $\gamma_{1}$ can be a convex path instead of a line segment.

In the definition 3 , during $\operatorname{mot}\left(A_{1}, A_{2}, A_{3}, \rho\right)$ movement, $A_{1}$ moves along the line segment $\gamma_{1}$. The line segment $\gamma_{1}$ can be replaced by a composition of two line segments in such a way that the path where $A_{1}$ belongs to is convex. See figure 3 .

In our algorithm, to reach $\mathrm{p}$ we have to apply $\operatorname{mot}\left(A_{i-1}, A_{i}, A_{i+1}, \rho\right)$ movement several times. At the end, possibly p can be reached by $A_{n}$ somewhere during one of the middle-joint-up or the front-link-forward. It means that algorithm stops before the last $\operatorname{mot}\left(A_{i-1}, A_{i}, A_{i+1}, \rho\right)$ movement is terminated. Such a movement is called partial-mot $\left(A_{i-1}, A_{i}, A_{i+1}, \rho\right)$ movement. This is a movement in according with the $\operatorname{mot}\left(A_{i-1}, A_{i}, A_{i+1}, \rho\right)$ movement, the movement stops somewhere during one of the middle-jointup or the front-link-forward motion in such a way that $A_{3}$ remains on $\gamma_{2}$.

\section{The reachability algorithm}

In this section, we study reachability in a region bounded by two squares in which the whole or a part of a side of one square coincides with a part of a side of the other. 
Assume $S_{1}$ and $S_{2}$ are two regions bounded by squares $\partial S_{1}$ and $\partial S_{2}$ with side lengths $s_{1}$ and $s_{2}$ respectively. Let squares $\partial S_{1}$ and $\partial S_{2}$ be attached via one side (the whole or a part of a side) and $\mathrm{S}=S_{1} \cup S_{2}$. Let $\Gamma=[0,1, \ldots n]$ be an n-linkage confined within $S_{1}$ (Figure 4-a). In the following theorem we explain how $A_{n}$, the end of $\Gamma$, can reach a point $\mathrm{p} \in S_{2}$.

Let $\rho$ be the line segment shared by $S_{1}$ and $S_{2}$ and let $v_{1}$ and $v_{2}$ be two end points of $\rho$, where $v_{1}$ is the farthest point of $\rho$ from $\mathrm{p}$ (Figure 4-b).

The following theorem presents sufficient condition for reachability of a given point in $\mathrm{S}$ by the end of a linkage confined within $\mathrm{S}$.

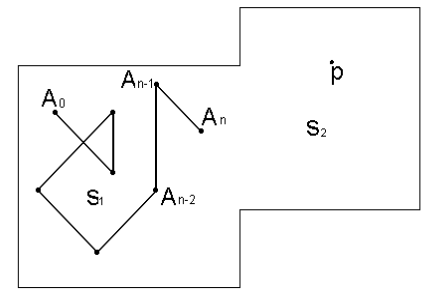

(a)

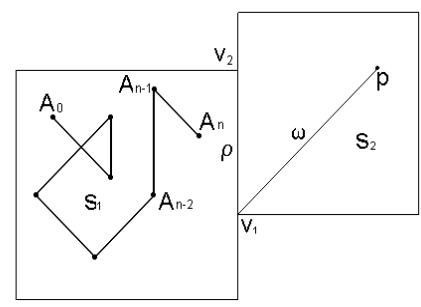

(b)

Figure 4: $\Gamma$ confined within $S_{1}$ and $p \in S_{2}$.

Theorem 4. Suppose $p \in S_{2}, \Gamma$ confined within $S_{1},\|\Gamma\|<\operatorname{Min}\left\{\frac{\sqrt{2}}{2} s_{1},\|\rho\|\right\}$, then with $\mathrm{O}\left(n^{2}\right)$ simple motions - in the worst case - p can be reached by $A_{n}$.

Proof: We introduce an algorithm to bring $A_{n}$ to $\mathrm{p}$ using $\mathrm{O}\left(n^{2}\right)$ simple motions, in the worst case.

Assume that $\omega$ is the line including $v_{1} \mathrm{p}$, and moving from $v_{2}$ to $v_{1}$ on the side of $\partial S_{1}$ which includes $v_{2}$ and $v_{1}$ is clockwise. At the beginning we bring $\Gamma$ to $\mathrm{ONF}$ in $S_{1}$. By theorem 1, this is done in $\mathrm{O}(\mathrm{n})$ simple motions. Without loss of generality we assume that $\Gamma$ is placed on $\partial S$ in counterclockwise order of indices of links'joints. Then $\Gamma$ is moved along $\partial S_{1}$ counterclockwise until $A_{n}$ reaches $v_{1}$. This can be done while no joint of $\Gamma$ leaves $\partial S_{1}$.

We consider two cases: $d\left(p, v_{1}\right) \geq\left\|A_{n-1} A_{n}\right\|$ and $d\left(p, v_{1}\right)<\left\|A_{n-1} A_{n}\right\|$. Case 1: $d\left(p, v_{1}\right) \geq\left\|A_{n-1} A_{n}\right\|$. The algorithm consists of three steps. In the first step $A_{n}$ is brought into $S_{2}$. In the second step $\Gamma$ is moved so that $\Gamma\left[0, k_{0}\right]$ takes ONF in $S_{1}\left(k_{0}\right.$ will be defined in step 2$), A_{k_{0}}$ coincides with $v_{1}$, and $\Gamma\left[k_{0}, n\right] \subset \omega$, and finally, in the last step $A_{n}$ reaches $\mathrm{p}$.

Step 1: Move $\Gamma$ along $\partial S_{1}$ counterclockwise until $A_{n-1}$ reaches $v_{1}$, because $\|\Gamma\|<\|\rho\|, A_{n}$ doesn't pass $v_{2}$, this takes $\mathrm{O}(\mathrm{n})$ (Figure 5 -a). Then rotate $A_{n}$ clockwise about $A_{n-1}=v_{1}$ toward $\omega$ until $A_{n}$ lies on $\omega$. If $d\left(p, v_{1}\right)=\left\|A_{n-1} A_{n}\right\|, A_{n}$ reaches $\mathrm{p}$ and we are done. If not, we pass to the second step. This step takes $\mathrm{O}(\mathrm{n})$.

Step 2: We define $k_{0}=\min \left\{\mathrm{k} \mid d\left(p, v_{1}\right) \geq \sum_{i=k+1}^{n} l_{i}\right\}$. Since $d\left(p, v_{1}\right) \geq$ $l_{n}$, then $k_{0} \leq n-1$. Suppose that, for $j>k_{0}, \Gamma[j, n] \subset \omega$ is straight, $A_{j}$ coincides with $v_{1}$, and $\Gamma[1, j]$ gets $\mathrm{ONF}$ in $S_{1}$, by using $\operatorname{mot}\left(A_{j-1}, A_{j}, A_{j+1}, \rho\right)$, 


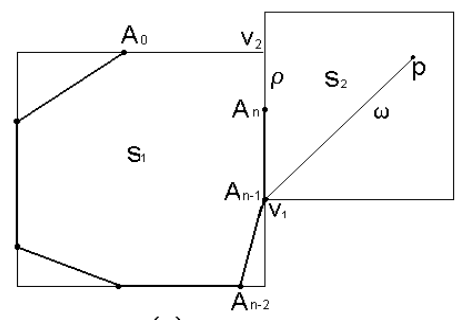

(a)

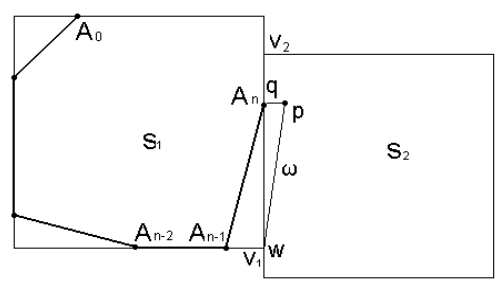

(b)

Figure 5: (a): $d\left(p, v_{1}\right)>\left\|A_{n-1} A_{n}\right\|,(b): d\left(p, v_{1}\right)<\left\|A_{n-1} A_{n}\right\|$ and $v_{1}=w$

$\Gamma$ is moved to a configuration in which $\Gamma[j-1, n] \subset \omega$ straightens, $A_{j-1}$ coincides with $v_{1}$, and $\Gamma[1, j-1]$ is in ONF in $S_{1}$.

By repeating this process, $\Gamma$ can move to a configuration in which, $\Gamma\left[1, k_{0}\right]$ gets ONF, $A_{k_{0}}$ coincides with $v_{1}$, and $\Gamma\left[k_{0}, n\right] \subset \omega$.

If $k_{0}>0$, since $\sum_{i=k_{0}}^{n} l_{i}>d\left(p, v_{1}\right)>\sum_{i=k_{0}+1}^{n} l_{i}, A_{n}$ reaches p during $\operatorname{mot}\left(A_{k_{0}-1}, A_{k_{0}}, A_{k_{0}+1}, \rho\right)$. Therefore we move $\Gamma$ according to partial$\operatorname{mot}\left(A_{k_{0}-1} A_{k_{0}}, A_{k_{0}+1}, \rho\right)$, depending on values of $\angle v_{2} v_{1} p, l_{k_{0}}$ and $d\left(p, v_{1}\right)$, $A_{n}$ reaches $\mathrm{p}$ during one of the middle-joint-up motion or the front-linkforward motion. This step takes $\mathrm{O}\left(k_{0} \mathrm{n}\right)$ and is $\mathrm{O}\left(n^{2}\right)$ in the worst case.

If $k_{0}=0, A_{n}$ doesn't reach p during this step and we pass to step 3 .

Step 3: In the case of $k_{0}=0$, i.e. $\sum_{i=1}^{n} l_{i}<d\left(p, v_{1}\right)$, by step2, $\Gamma$ may move to a configuration in which, $A_{0}$ coincides with $v_{1}$ and $\Gamma \subset \omega$ straightens. It is enough to move $\Gamma$ along $\omega$ toward p until $A_{n}$ reaches p. This step takes $\mathrm{O}(1)$.

Case 2: $d\left(p, v_{1}\right)<\left\|A_{n-1} A_{n}\right\|$. Assume that $\omega$ intersects $\partial S_{1}$ at $\mathrm{w}$ (it is possible that $\mathrm{w}$ may coincides with $v_{1}$ (Figure 5 -b)). Let the circle $C\left(v_{1},\left\|p v_{1}\right\|\right)$ intersect $v_{1} v_{2}$ at $\mathrm{q}$. To reach $\mathrm{p}$, move $\Gamma$ counterclockwise along $\partial S_{1}$ until $A_{n}$ reaches q. Depending on the position of $A_{n-1}$ on $\partial S_{1}$ one of the three following subcases occurs.

Subcase 2.1: $A_{n-1}$ resides on the side of $\partial S_{1}$ containing $v_{1} v_{2}$. In this situation $v_{1}$ belongs to the link $A_{n-1} A_{n}$ and $C\left(p, l_{n}\right)$ intersects the line segment $\omega$ at point g. Rotate $A_{n-1} A_{n}$ clockwise about $v_{1}$ toward p. Because $\|\Gamma\|<\frac{\sqrt{2}}{2} s_{1}, C\left(g, l_{n-1}\right)$ cannot contain $S_{1}$ i.e. $A_{n-2}$ does not need to exit $S_{1}$. Continue rotation until $A_{n-1}$ reaches g and $A_{n}$ reaches p. During rotation, $A_{n-1}$ exits $\partial S_{1}$ and if $C\left(g, l_{n-1}\right)$ intersects $\partial S_{1}, A_{n-2}$ can be stayed on $\partial S_{1}$ and $\Gamma[0 \ldots n-2]$ remains in ONF (Figure 6-a).

Otherwise if $C\left(g, l_{n-1}\right)$ does not intersect $\partial S_{1}$, consider the largest $0<k_{0}$ in such a way $C\left(g, l_{n-1} \ldots+l_{k_{0}}\right)$ intersects $\partial S_{1}$, otherwise let $k_{0}=1$. During rotation we let $A_{n-1}, \ldots, A_{k_{0}}$ exit $\partial S_{1}$ while making $\alpha_{n-1}=\ldots=\alpha_{k_{0}+1}=\pi$, keeping $\Gamma\left[k_{0} \ldots n-1\right]$ straight and remaining $\Gamma\left[0 \ldots k_{0}\right]$ in ONF.

Subcase 2.2: $A_{n-1}$ resides on the side of $\partial S_{1}$ adjacent to the side containing $v_{1} v_{2}$, and $\omega$ intersects link $A_{n} A_{n-1}$. To reach p, first fix $\Gamma[0,1, \ldots n-1]$ and rotate $A_{n-1} A_{n}$ about $A_{n-1}$ toward p until link $A_{n-1} A_{n}$ reaches $v_{1}$. Then rotate $A_{n-1} A_{n}$ about $v_{1}$ toward $\omega$ until $A_{n}$ hits $\omega$. During rotation $A_{n}$ does not hit $\partial S_{1}$. Finally slip $A_{n-1} A_{n}$ on $\omega$ until $A_{n}$ reaches p. During the move- 


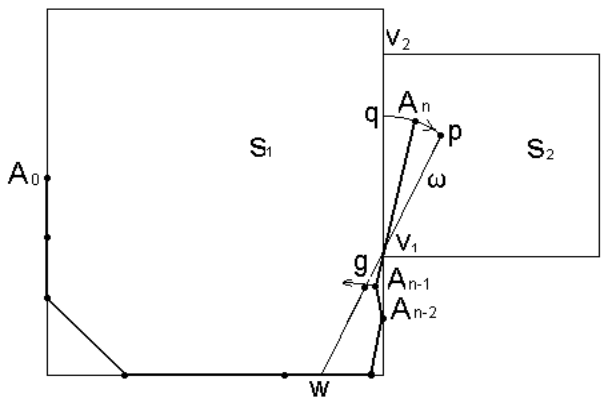

(a)

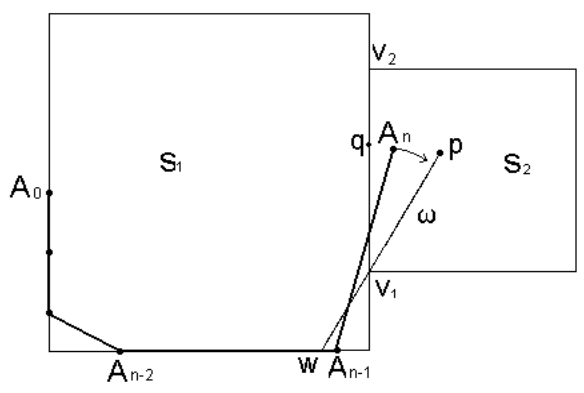

(b)

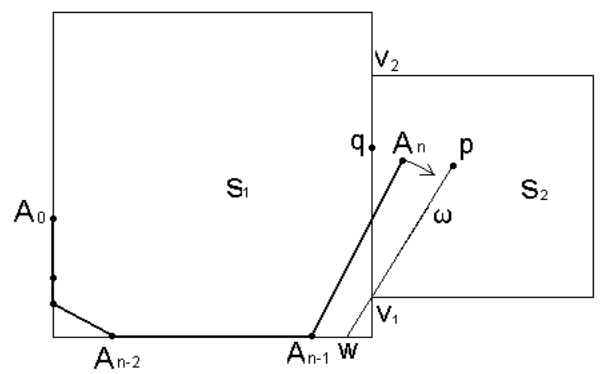

(c)

Figure 6: (a): $A_{n-1}$ belongs to the same edge as $v_{1},(b): A_{n}$ and $A_{n-1}$ are in both sides of $\omega,(c): A_{n}$ and $A_{n-1}$ are in the same side of $\omega$

ment, one of the possibilities similar to the previous situation will happen, which can be treated accordingly (Figure 6-b).

Subcase 2.3: Like case 2.2, but $\omega$ does not intersect link $A_{n} A_{n-1}$. Suppose that $C\left(p, l_{n}\right)$ intersects $\partial S_{1}$ at g. i.e. p is visible from g. To reach p, first fix $\Gamma[0,1, \ldots n-1]$ and rotate $A_{n-1} A_{n}$ about $A_{n-1}$ toward $\omega$ until $A_{n}$ reaches $\omega$. Then, move $A_{n}$ along $\omega$ toward p. During movement $\Gamma[0,1, \ldots n-$ 1] does not exit $\partial S_{1}$ and $A_{n}$ gets to p while $A_{n-1}$ reaches g. Refer to Figure 6-c.

Each of these subcases takes $\mathrm{O}(\mathrm{n})$.

\section{References}

[1] J. Hopcroft, D. Joseph and S. Whitesides. Movement problems for 2dimensional linkages. SIAM J. Compt., 13: pp. 610-629, 1984.

[2] J. Hopcroft, D. Joseph and S. Whitesides. On the movement of robot arms in 2-dimensional bounded regions. SIAM J. Compt., 14: pp. 315333, 1985.

[3] D. Joseph and W.H. Plantings. On the complexity of reachability and motion planing questions. Proc. of the symposium on computational geometry. ACM, June 1985. 
[4] V. Kantabutra. Motions of a short-linked robot arm in a square. Discrete and Compt. Geom., 7:pp. 69-76, 1992.

[5] V. Kantabutra. Reaching a point with an unanchored robot arm in a square. International jou. of comp. geo. \& app., 7(6):pp. 539-549, 1997.

[6] W.J. Lenhart and S.H. Whitesides. Reconfiguration using line tracking motions. Proc. 4th Canadian Conf. on computational geometry, pp. 198-203, 1992.

[7] M. van Krevel, J. Snoeyink and S. Whitesides. Folding rulers inside triangles. Discrete Compt. Geom., 15:pp. 265-285, 1996. 